\title{
AUTOMATED SEGMENTATION OF THE RIGHT HEART USING AN OPTIMIZED SHELLS AND SPHERES ALGORITHM
}

\author{
C. Aaron Cois ${ }^{1}$, Ken Rockot $^{1}$, John Galeotti ${ }^{2}$, Robert Tamburo ${ }^{1}$, Danielle Gottlieb ${ }^{3}$ \\ John E. Mayer, Jr. ${ }^{3}$, Andrew Powell ${ }^{3}$, Michael Sacks ${ }^{1}$, George Stetten ${ }^{1,2}$ \\ Department of Bioengineering, University of Pittsburgh ${ }^{1}$ \\ The Robotics Institute, Carnegie Mellon University ${ }^{2} \quad$ Children's Hospital, Boston ${ }^{3}$
}

\begin{abstract}
We have developed a novel framework for medical image analysis, known as Shells and Spheres. This framework utilizes spherical operators of variable radius centered at each image pixel and sized to reach, but not cross, the nearest object boundary. Statistical population tests are performed on adjacent spheres to compare image regions across boundaries. Previously, our framework was applied to segmentation of cardiac $\mathrm{CT}$ data with promising results. In this paper, we present a more accurate and versatile system by optimizing algorithm parameters for a particular data set to maximize agreement to manual segmentations. We perform parameter optimization on a selected 2D slice from a 3D image data set, generating effective parameters for 3D segmentation in practical computational time. Details of this approach are given, along with a validated application to cardiac MR data.
\end{abstract}

\section{INTRODUCTION}

Medical images provide the means for clinicians to obtain a wide variety of in-situ structural and functional data on which to base diagnoses in a non-invasive manner, and as such provide an extremely powerful clinical tool. The ability to visualize shape, location, deformation, and other structural information pertaining to various anatomical structures is useful in surgical planning, diagnosis, and post-surgical evaluation, as well as many avenues of medical research. However, interpreting imaging data can be difficult, and delineating specific anatomical structures and quantifying particular geometric values usually requires highly trained radiologists and technicians.

Segmentation is one of the most commonly desired procedures in medical image analysis. The process of segmenting, or delineating, a specific anatomical structure within an image is a complex procedure, hampered by image noise, blurred boundaries, limitations in data resolution, and ultimately by subjectivity in the very definition of objects within medical images. Processing 3D data, instead of 2D slices, may introduce other problems such as anisotropy in resolution, when in-slice pixel resolution is greater than between-slice spacing.

Manual segmentation is still the most common clinical practice [2], though it is time-consuming (especially for 3D images) and requires a great deal of anatomical knowledge and clinical expertise on the part of the user. In addition to being slow and labor-intensive, manual segmentation is prone to significant variation between users due to the subjectivity of image interpretation.

We have developed a novel framework, called Shells and Spheres, for automated medical image analysis and segmentation [1]. We have obtained promising results using a segmentation algorithm based on this framework, which employs variable-scaled spherical operators as representative populations of image objects. We will present background on the basic concepts and statistics used by the Shells and Spheres framework in the next section. In our previous research [1], parameters were chosen manually and ad hoc to yield results that were qualitatively pleasing. In this paper, we describe a more rigorous parameter optimization procedure to maximize segmentation accuracy for a given data set. Results and validation data for our parameter optimization technique and resulting automated 3D segmentations of the right heart generated from MR data will be presented.

\section{BACKGROUND: SHELLS AND SPHERES}

The framework of Shells and Spheres is based on a construct called a sphere map. A sphere map is a set of spheres, exactly one sphere centered at each image pixel, whose radii can be individually adjusted. Calculations denoted as Variable-Scale Statistics (VSS) are performed on populations of pixels within spheres, as well as populations of adjacent and overlapping spheres. Memory and computation requirements are kept reasonable by storing only a relatively small, fixed number of VSS at each pixel, many of which can be updated incrementally as spheres grow or shrink. The ultimate goal of radii adjustment is to produce a sphere map in which each sphere is as large as possible without crossing a boundary. Therefore, once properly adjusted, the spheres' radii are equivalent to what is commonly known as a distance map [3]. Though the task is trivial in binary images, where definitive boundaries are known, it presents a challenge when boundaries are difficult to determine due to noise and tissue inhomogeneity. The Shells and Spheres approach is well suited to this challenge, as it makes use of large pixel populations defined by maximally sized spheres for its region-defining statistics, though it is understood that the correctness of segmentation in real images is ultimately subjective.

Many conventional methods for image processing consider a region of fixed size and shape, usually referred to as a kernel, especially when used for convolution. Other common approaches define dynamic regions adjoining boundaries using deformable contours [4] or level sets [5]. Our approach, instead, uses a set of spheres whose individual radii are optimized using VSS operators to achieve maximum discrimination between image regions. Not only can such spheres provide populations that are accurate and versatile for boundary detection and region representation, but those spheres that touch at least two boundaries are also medial, as classically defined by Blum [6], providing a basis for medial feature extraction. And perhaps most importantly, unlike Gaussian blurring, 
commonly used in multi-scale analysis [7, 8], Shells and Spheres preserves sharp boundaries with increasing scale.

\subsection{Algorithm 1}

The concepts of Shells and Spheres, VSS, and their potential applications to image analysis have been developed in detail in [1], with an emphasis on segmentation. In that work, a particular algorithm utilizing the framework was developed for segmenting the aortic arch in contrast-enhanced CT data. We will refer to that algorithm here as Algorithm 1, since a wide variety of diverse algorithms may be created for segmentation, as well as for other image analysis tasks, using Shells and Spheres. A brief overview of Algorithm 1 will now be given to introduce the background concepts of the research presented in this paper.

The Shells and Spheres Algorithm 1 takes the form of a six step process, with steps 1-4 optimizing the sphere map, step 5 finding medial pixels, and step 6 producing an object segmentation.

Step 1 creates an initial approximation of the sphere map, from which acceptable statistical values can be obtained for use in subsequent steps. This approximation is based on determining probable boundaries by detecting an increasing first-order moment of intensity within each sphere as it grows. A persistent moment is assumed to be a probable boundary, past which sphere growth is limited.

Step 2 utilizes the pronounced discrepancy in variance between spheres that have incorrectly grown across boundaries and those that have not to shrink incorrect spheres, placing them correctly within their appropriate image objects. The discrepancy is large enough to permit the use of a global variance measure, $\beta_{s}$, which will be discussed further below.

Step 3 introduces specialized boundary indicators, known as outposts, to the image based on boundary information between spheres in adjacent objects. These outposts influence the radii of nearby spheres, resizing them to adhere to the consensus of boundary information.

Step 4 revisits variance using the updated sphere map, which is more accurate and therefore capable of delivering a better estimate of global variance. The new estimate, based on parameter $\beta_{g}$, is used to grow spheres fully within the object boundaries. This step increases the accuracy of the sphere map with respect to the image.

Step 5 identifies medial pixels, i.e. those whose spheres touch at least two boundaries.

Step 6, given a manually selected medial seed point, connects neighboring medial pixels and combines their corresponding spheres to produce a segmentation.

This algorithm has been enhanced in our current research to include a system for optimizing the parameters $\beta_{s}$ and $\beta_{g}$ based on manually segmented data, as will be presented in the next section. This has permitted the application of the algorithm to more challenging data, MR images of the right cardiac ventricle.

\section{PARAMETER OPTIMIZATION}

In our initial application of Algorithm 1 to cardiac segmentation, algorithm parameters were manually derived for the particular data, limiting the systems accuracy and versatility. For example, when the parameters used to successfully segment the aortic arch in [1] were applied to our RVOT data, the automated segmentation failed completely because the parameters are dependent on imaging modality and target structure. To improve segmentation accuracy and adapt our algorithm to new data sets, an optimization method was developed for two key algorithm parameters discussed above, $\beta_{s}$ and $\beta_{g}$,

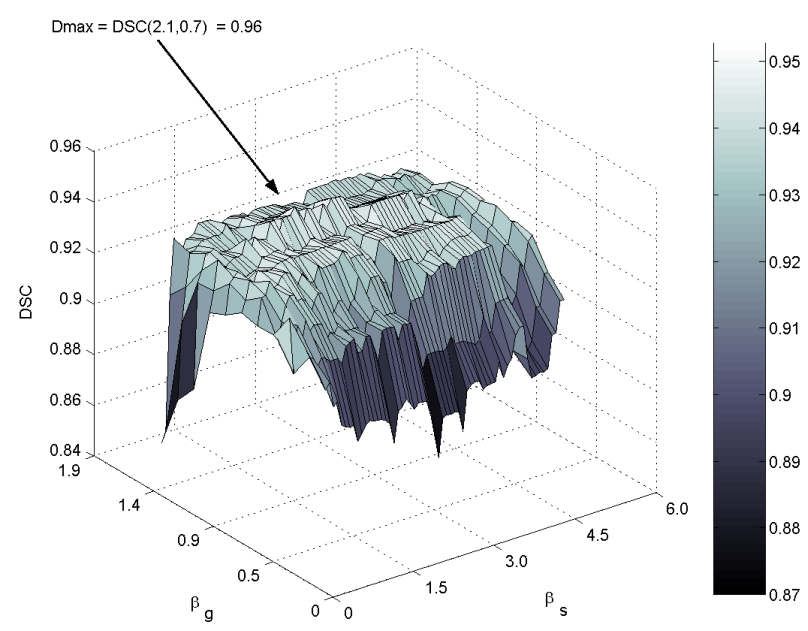

Fig. 1. Example Dice Similarity Coefficient (DSC) curve produced by a $2 \mathrm{D}$ parameter optimization with $\beta_{s} \in 0.0: 6.0, \beta_{g} \in 0.0: 1.9$, $j=0.1$. The optimal DSC value 0.96 , produced by the parameter set $\left(\beta_{s}=2.1, \beta_{g}=0.7\right)$, is indicated with an arrow.

which control variance thresholds regulating sphere shrinkage and growth, respectively. Other parameters, believed to have significantly less impact on the resulting segmentation, were left at the default values given in [1]. The parameters $\beta_{s}$ and $\beta_{g}$ were optimized to maximize the resulting agreement, defined by the Dice Similarity Coefficient (DSC) [9], between the automated segmentation and a gold standard manual segmentation. The DSC, briefly, is the ratio of twice the number of intersecting pixels between the two objects and the combined number of pixels within both objects. This coefficient is bounded by 0 and 1 , with a value of 0 resulting from no intersection and 1 resulting from complete overlap. This value will be represented as

$$
D=D S C\left(\beta_{s}, \beta_{g}\right)
$$

for given values of $\beta_{s}$ and $\beta_{g}$.

A brute-force optimization approach, testing every combination of parameter values within given ranges at a given increment $j$, was applied to deduce the optimal parameter values for a given data set. The optimal parameter values are defined by the maximal DSC, as

$$
D_{\text {max }}=\underset{\beta_{s} \in \beta_{s i}: \beta_{s f}, \beta_{g} \in \beta_{g i}: \beta_{g f}}{\operatorname{argmax}}\left(D S C\left(\beta_{s}, \beta_{g}\right)\right) .
$$

The value of $\beta_{s}$ was discretely sampled at intervals of $j$ over a range $\beta_{s i}: \beta_{s f}$, where $\beta_{s i}$ indicates initial $\beta_{s}$ and $\beta_{s f}$ indicates final $\beta_{s}$. Similarly, $\beta_{s}$ was over a range of $\beta_{g i}: \beta_{g f}$. This brute-force data gathering approach produces a DSC curve as seen in Figure 1. The maximal DSC value of 0.96 on the upper ridge of the curve, as indicated by an arrow, corresponds, in this case, to the parameter values $\beta_{s}=2.1$ and $\beta_{g}=0.7$.

Though effective at producing parameters that resulted in accurate segmentations, this optimization technique was computationally time-consuming. For example, the 2D image used to produce the optimization in Figure 1 was a 256x256 pixel MRI image. A segmentation of this single 2D image via our Shells and Spheres algorithm 


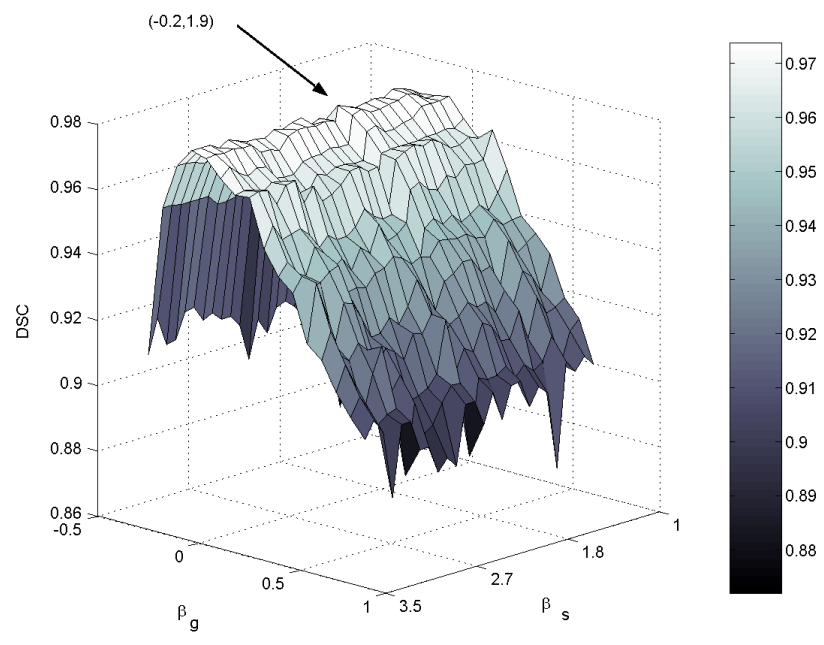

Fig. 2. DSC curve produced by a $2 \mathrm{D}$ parameter optimization with $\beta_{s} \in 1.0: 3.5, \beta_{g} \in-0.5: 1.0, i=0.1$. The optimal DSC, 0.97, is indicated with an arrow at values $\beta_{s}=1.9, \beta_{g}=-0.2$ )

took approximately 1 minute. The curve shown in Figure 1 represents 61 values of $\beta_{s}$ and 20 values of $\beta_{g}$, with each combination of parameter values producing a different segmentation. Thus, it required 1,220 analyses to create, or 20.3 hours of computation. While search-based optimization techniques are being explored to replace the brute-force approach, the fact that a single segmentation of a 3D data set can take hours on its own indicates that even search-based optimization would be computationally untenable in 3D data.

To allow for effective parameter optimization of 3D data, a method of utilizing 2D slices for 3D parameter optimization has been developed. Optimization of parameters for automated 3D analysis and segmentation can be achieved by optimizing the parameters on a $2 \mathrm{D}$ slice from the target data set, rather than the full 3D data set, and then applying the optimal parameters thus determined to 3D analysis of the full data set. This technique not only makes the optimization computationally tenable, but also reduces human requirements by only requiring a manual segmentation of a single $2 \mathrm{D}$ slice, rather than a full 3D segmentation. We will present results and validation of 3D segmentations utilizing this parameter optimization technique in the next section.

\section{RESULTS AND VALIDATION}

To determine the effectiveness of our parameter optimization technique on 3D analysis and segmentation, a 3D segmentation of the right heart was performed on an MR scan of an ovine subject, seen on the left side of Figure 5. Images were obtained in vivo, with a 1.5T Philips Intera Achieva MR scanner using white blood imaging (T2 with SPIR, respiratory navigator gating) with the following parameters: FOV 350; TR 3.5; TE 1.8; flip angle 55;RFOV 70\%; NSA 1; TFE factor 14; slice thickness 1.4/0.7; matrix 224/256 x 224.

A manually selected 2D slice, shown in Figure 3, was extracted from the $3 \mathrm{D}$ data set, manually segmented, and used to perform a parameter optimization, producing the DSC curve shown in Figure 2. It should be noted that this is a different curve than that shown in Figure 1. The data in Figure 2 was derived from the ovine cardiac MR

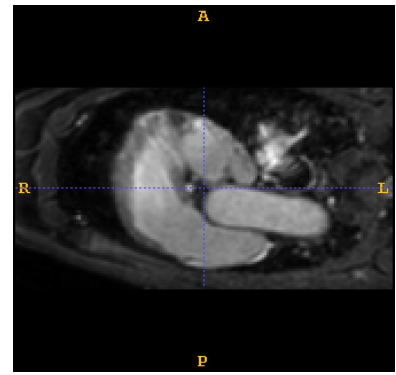

(a)

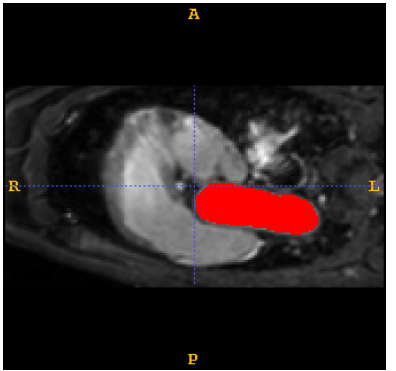

(b)
Fig. 3. (a) 2D slice of MR cardiac data and (b) manual segmentation (red or dark) used for parameter optimization in the 3D segmentation study.

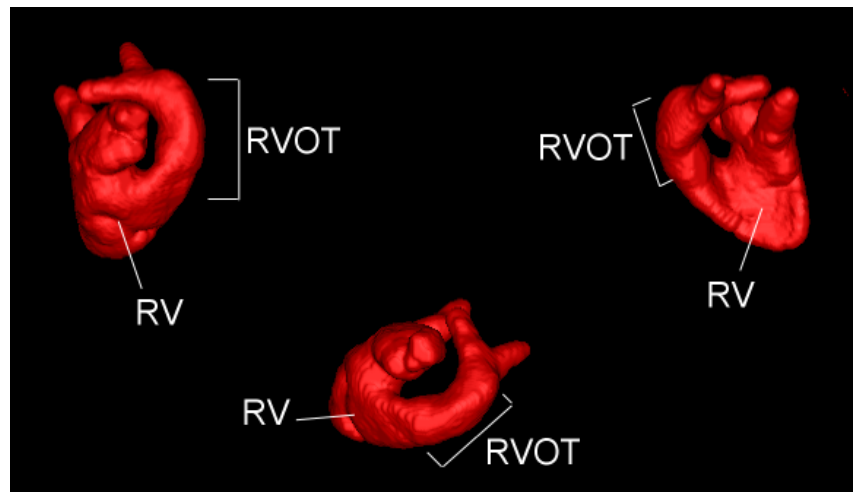

Fig. 4. Surface model of the automated segmentation of the right heart with the RV and RVOT labeled, shown from three different perspectives.

image shown in Figure 5. This slice, and the selected object within it (the aortic arch), were chosen because the thin boundaries between adjoining larger objects simulated conditions for the 3D segmentation of the right ventricle adjoining other cardiac structures.

The optimization yielded values of $\beta_{s}=1.9$ and $\beta_{g}=-0.2$ for this data set, which produced a DSC of 0.97. This set of parameters, determined on a 2D slice, enabled our Shells and Spheres algorithm to produce a 3D segmentation of the right heart, shown anatomically labeled in multiple orientations in Figure 4 and overlaid on the MR data in Figure 5.

To test the accuracy of the 3D segmentation, a validation study was conducted to compare it to three manual segmentations. Each segmentation was produced by a different user. Table 1 shows the DSC values for our automated segmentation compared to the manual segmentations, as well as the DSC values for the manual segmentations compared to each other. It can be seen that our automated segmentation matched the manual segmentations with a DSC between 0.83 and 0.86 . It should be noted that a DSC of 0.70 is considered excellent agreement in the literature [10], although the definition of sufficient accuracy is, of course, specific to the application. While the manual segmentations produced slightly higher agreement with each other than with the automated segmentation, it is believed that a significant portion of this discrepancy is due to the difficulty defining 


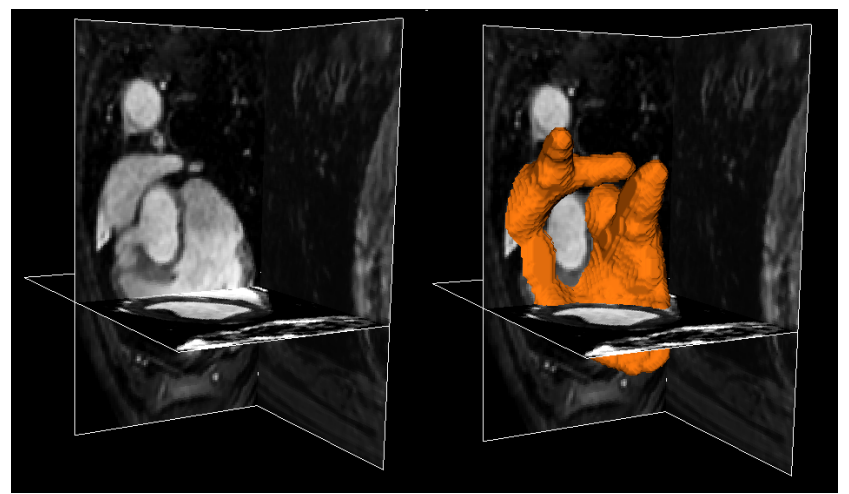

Fig. 5. Left: The ovine cardiac MR data set used in our segmentation study. Right: A surface model of the automated segmentation produced by our Shells and Spheres algorithm overlaid on the MR data.

Table 1. Table of DSC values comparing segmentations produced by 3 independent subjects and our Shells and Spheres (S\&S) algorithm.

\begin{tabular}{|c|c|c|c|}
\hline & Subject 1 & Subject 2 & Subject 3 \\
\hline S\&S & 0.83 & 0.84 & 0.86 \\
\hline Subject 1 & $*$ & 0.91 & 0.88 \\
\hline Subject 2 & $*$ & $*$ & 0.89 \\
\hline
\end{tabular}

the extent of the "right heart" along the continuum of the circulatory system. Subject 3 elected to include less of the branching vasculature connected to the main cardiac structures, which led to greater agreement with the automated segmentation and less agreement with the other manual segmentations. Despite the variation in manual segmentations, our system still demonstrated reliable segmentation results.

\section{CONCLUSIONS}

This study validated the effectiveness of Shells and Spheres Algorithm 1 for segmentation of the right heart in cardiac MR data. The right heart provides additional challenges for segmentation absent in our previous work with the aorta, in particular, the presence of several large, adjacent structures of similar intensity. The study confirmed our expectation that algorithm parameters could be optimized for automated 3D segmentation based on a 2D manual segmentation of a slice from the same data set. Pilot optimization on a $2 \mathrm{D}$ slice to obtain parameters for $3 \mathrm{D}$ analysis could be a very practical means of finding optimal parameters for a particular imaging modality and anatomical target, or even a particular clinical 3D scan. This finding increases the power and versatility of our analysis and segmentation framework by providing an effective method for tailoring the algorithm parameters to maximize the accuracy of segmentation and provides evidence for the validity of the Shell and Spheres framework.

\section{ACKNOWLEDGEMENTS}

- Supported by a contract from the National Library of Medicine for the development of the Insight Toolkit (ITK) with which this work was implemented.

- Supported by Sanjeev Schroff and the NIH through the Cardiovascular Bioengineering Training Program, NIH-T32-HL76124.

- Supported in part by a NSF Graduate Student Fellowship.

- Supported in part by NIH/NHLBI R01 068816.

\section{REFERENCES}

[1] C. Aaron Cois, "Shells and spheres: A novel framework for variable-scale statistical image analysis," M.S. thesis, Department of Computer Science, University of Pittsburgh, Pittsburgh, PA, August 2006.

[2] Paul A. Yushkevich, Joseph Piven, Heather Cody, Sean Ho, James C. Gee, and Guido Gerig, "User-guided level set segmentation of anatomical structures with itk-snap," IJ - 2005 MICCAI Open-Source Workshop, July 2005.

[3] Per Erik Danielsson, "Euclidean distance mapping," Computer Graphics and Image Processing, vol. 14, no. 3, pp. 227-248, Nov. 1980.

[4] M. Kass, A. Witkin, and D. Terzopoulos, "Snakes: Active contour models," International Journal of Computer Vision, vol. 1, no. 4, pp. 321-331, 1988.

[5] James Albert Sethian, Level set methods: Evolving interfaces in geometry, fluid mechanics, computer vision, and materials science, Number 3 in Cambridge monographs on applied and computational mathematics. Cambridge University Press, Cambridge, U.K., 1996, 218 pages.

[6] H. Blum and R. N. Nagel, "Shape description using weighted symmetric axis features," Pattern Recognition, 10, pp. 167180, 1978.

[7] Tony Lindeberg, Discrete Scale-Space Theory and the ScaleSpace Primal Sketch, Ph.D. thesis, Department of Numerical Analysis and Computer Science, KTH, Stockholm, Sweden, May 1991.

[8] Tony Lindeberg, Scale-Space Theory in Computer Vision, Kluwer Academic Publishers, Dordrecht, Netherlands, 1994.

[9] L. R. Dice, "Measures of the amount of ecologic association between species," Journal of Ecology, vol. 26, pp. 297-302, 1945.

[10] A. Zijdenbos, B. Dawant, and R. Marjolin, "Morphometric analysis of white matter lesions in mr images: Methods and validation," IEEE TMI, vol. 13(4), pp. 716-724, 1994. 